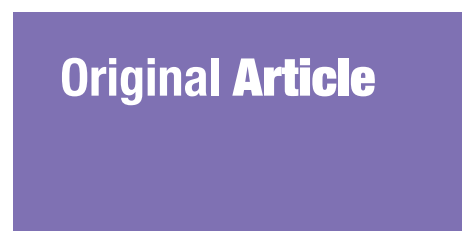

Submitted: 25 May 2017 Accepted: 10 Sep 2017 Online: 29 Dec 2017

\section{Construction and Cloning of Reporter- Tagged Replicon cDNA for an In Vitro Replication Study of Murine Norovirus-1 (MNV-1)}

\author{
Muhammad Khairi Ahmad ${ }^{1,2}$, Yasser M Tabana ${ }^{1}$, MowaffaQ \\ Adam Ahmed', Doblin Anak Sandai', RafeEzul Mohamed ${ }^{3}$, \\ Ida Shazrina Ismail ${ }^{3}$, Nurulisa Zulkiflie ${ }^{2}$, Muhammad Amir \\ Yunus $^{1}$ \\ 1 Infectomics Cluster, Advanced Medical \& Dental Institute, Universiti Sains \\ Malaysia, Bertam, 13200 Kepala Batas, Pulau Pinang, Malaysia \\ 2 Oncological \& Radiological Science Cluster, Advanced Medical \& Dental \\ Institute, Universiti Sains Malaysia, Bertam, 13200 Kepala Batas, \\ Pulau Pinang, Malaysia \\ 3 Regenerative Medicine Cluster, Advanced Medical \& Dental Institute, \\ Universiti Sains Malaysia, Bertam, 13200 Kepala Batas, Pulau Pinang, \\ Malaysia
}

To cite this article: Muhammad Khairi A, Yasser MT, Mowaffaq AA, Doblin AS, Rafeezul M, Ida Shazrina I, Nurulisa Z, Muhammad Amir Y. Construction and cloning of reporter-tagged replicon cDNA for an in vitro replication study of murine norovirus-1 (MNV-1). Malays J Med Sci. 2017;24(6):29-38. https://doi.org/10.21315/mjms2017.24.6.4

To link to this article: https://doi.org/10.21315/mjms2017.24.6.4

\begin{abstract}
Background: A norovirus maintains its viability, infectivity and virulence by its ability to replicate. However, the biological mechanisms of the process remain to be explored. In this work, the NanoLuc ${ }^{\mathrm{TM}}$ Luciferase gene was used to develop a reporter-tagged replicon system to study norovirus replication.

Methods: The NanoLuc ${ }^{\mathrm{TM}}$ Luciferase reporter protein was engineered to be expressed as a fusion protein for MNV-1 minor capsid protein, VP2. The foot-and-mouth disease virus 2A (FMDV2A) sequence was inserted between the 3'end of the reporter gene and the VP2 start sequence to allow co-translational 'cleavage' of fusion proteins during intracellular transcript expression. Amplification of the fusion gene was performed using a series of standard and overlapping polymerase chain reactions. The resulting amplicon was then cloned into three readily available backbones of MNV-1 cDNA clones.

Results: Restriction enzyme analysis indicated that the NanoLucTM Luciferase gene was successfully inserted into the parental MNV-1 cDNA clone. The insertion was further confirmed by using DNA sequencing.

Conclusion: NanoLuc ${ }^{\mathrm{TM}}$ Luciferase-tagged MNV-1 cDNA clones were successfully engineered. Such clones can be exploited to develop robust experimental assays for in vitro assessments of viral RNA replication.
\end{abstract}

Keywords: luciferases, foot-and-mouth disease virus, norovirus, capsid proteins, virus replication, replicon, restriction mapping 


\section{Introduction}

Human norovirus (HuNV) is one of the leading causes of non-bacterial acute gastroenteritis worldwide and is responsible for a large number of epidemics annually (1). From a biological stand point, HuNVs are members of the Caliciviridae family i.e., they are structurally small viruses with a positive-sense RNA genome (2). The clinical manifestations of HuNV infection include acute and rapidonset diarrhea, nausea, vomiting, headache, fever, anorexia and malaise. Aside from these classical symptoms, the infection can lead to severe clinical outcomes, such as necrotising enterocolitis, seizures in infants, encephalopathy, pneumatosis intestinalis and disseminated intravascular coagulation (3-9). In a hospital setting, HuNV risk group comprises of patients with underlying conditions like cardiovascular diseases, patients with a kidney transplant, and cancer patients under immunosuppressive therapy (10-12). Despite its high prevalence, many of the biological functions and properties of HuNV are not properly understood due to the lack of an efficient cell culture system or a smallanimal model that may be used to study human norovirus (13). However, in 2003, a model based on mouse norovirus, namely murine norovirus (MNV), was introduced as a useful system to study noroviruses' biology and pathogenesis as MNV is able to replicate in cell culture and in small animals $(13,14)$.

From a molecular perspective, MNV shares many genetic and biochemical properties with human norovirus in terms of size, shape and buoyant density (15). HuNV has three open reading frames (ORF 1-3), whereas MNV contains four open reading frames (ORF 1-4). ORF1 encodes a large polyprotein which are coand post-translationally cleaved into six mature non-structural proteins, including a RNAdependent RNA polymerase (RdRp). Moreover, ORF2 encodes a major capsid protein (VP1); and $\mathrm{ORF}_{3}$ encodes a minor capsid protein (VP2) (16). The recently discovered $\mathrm{ORF}_{4}$ in $\mathrm{MNV}$ encodes virulence factor 1 (VF1) (17). Besides, in all noroviruses, the viral protein genomelinked (VPg) is attached at the 5' end, while the polyadenosine (poly-A) sequence is at the 3' end. All of these structural properties are vital for noroviruses translation, replication and pathogenesis (17-20).

A large number of replicon systems have been developed in the past with different reporter genes to study viruses with the main interest being the replication mechanisms as these are the key determinants of virulence and infectivity. Previous reporter-tagged replicon systems developed to study multiple viruses include Renilla Luciferase, Firefly Luciferase, mCherry-tagged replicon system and others (21, 22). In this study, we developed a NanoLuc ${ }^{\mathrm{TM}}$ Luciferase-tagged replicon system specifically to study MNV-1. Many aspects of viral replication and translation were considered when designing the replicon system to achieve functionality and examine translational effectiveness in accordance with the biological mechanism that takes place in the virus during the characterisation process.

\section{Material and Methods}

This study consisted of two major parts. The first part focused on the designing and construction of an MNV-1 based NanoLuc ${ }^{\mathrm{TM}}$ Luciferase-tagged replicon, whereas the second part had to do with the cloning of the constructed reporter gene into MNV-1 cDNA clones. The clones used were $\mathrm{pT}^{\mathrm{T}}$ : MNV 3 'Rz i.e., they were readily available in three backbone structures: (1) the wild-type (wt), (2) the NS7 frameshift mutant (F/S) and (3) the NS7 active site mutant $(\Delta \mathrm{NS} 7)$. The wt plasmid served as a replicationcompetent cDNA clone, of which the F/S and the $\Delta \mathrm{NS} 7$ plasmids were derived with mutations in the NS7 region of MNV-1 genome that encodes a viral RNA replicative enzyme. The derivative plasmids served as replication-deficient cDNA clones. All of the MNV-1 cDNA clones were supplied free-of-charge by the Ian Goodfellow's Laboratory at the University of Cambridge, UK.

\section{Construction of NanoLuc ${ }^{\mathrm{TM}}$ Luciferase- Tagged Replicon of MNV-1}

\section{Designing primers for polymerase chain reaction ( $P C R$ )}

Forward and reverse primers were designed and used in three PCR and two overlappingextension PCR (OE-PCR) amplification reactions (Table 1). All primers were purchased from First BASE Sdn Bhd, Malaysia. A partial sequence of the NanoLuc ${ }^{\mathrm{TM}}$ Luciferase (secNluc) gene was introduced to the primers (underlined) as described in Table 1. A forward primer, 5052F, and $\mathrm{A}$ reverse primer, $\mathrm{AY} 1$, were used in the first PCR reactions. These primers were later substituted with $\mathrm{AY} 2$ and $\mathrm{AY}_{3}$, respectively, in the second PCR amplification. As to the 
Table 1. List of primers and their sequences

\begin{tabular}{|c|c|c|}
\hline Primer & Sequence & Description \\
\hline $5052 \mathrm{~F}$ & $\begin{array}{l}\text { GTGAATGAGGATGAGTGATGGCGCAGCGCCAAAA } \\
\text { GCCAATGG }\end{array}$ & MNV-1 sequencing forward primer \\
\hline AY1 & $\begin{array}{l}\text { GCGCTTGTGGAGAAGGAGTTCATTATTGTTTGAGC } \\
\text { ATTCGGCCT }\end{array}$ & $\begin{array}{l}\text { Reverse primer to amplify MNV-1 Cterm VP1 } \\
+ \text { ATG + Nterm secNluc (pNL1.3) }\end{array}$ \\
\hline AY2 & $\begin{array}{l}\text { GGCAACAGGCCGAATGCTCAAACAATAATGAACTC } \\
\text { TTTCTCCACAAGCGC }\end{array}$ & $\begin{array}{l}\text { Forward primer for secNluc2A + VP2 } \\
\text { construction-overlapping with } \mathrm{AY} 1\end{array}$ \\
\hline $\mathrm{AY}_{3}$ & $\begin{array}{l}\text { CGCTCCAAAAAGAGCACCTGCAGGCCCAGGGTTGG } \\
\text { ACTCGACGTCTCCCGCAAGCTTAAGAAGGTCAAAA } \\
\text { TTCAACAGCGCCAGAATGCGTTCGCACAGC }\end{array}$ & $\begin{array}{l}\text { Reverse primer Cterm secNluc }+2 \text { Asequence } \\
+ \text { Nterm VP2 }\end{array}$ \\
\hline $\mathrm{AY}_{4}$ & $\begin{array}{l}\text { GACGTCGAGTCCAACCCTGGGCCTGCAGGTGCTC } \\
\text { TTTTTGGAGCGATTGG }\end{array}$ & Forward : FMDV2A + NtermVP2 \\
\hline $\mathrm{AY}_{5}$ & $\begin{array}{l}\text { GGCCGGCGCTAGCTTTTTTTTTTTTTTTTTTTTTT } \\
\text { TTTTTAAAATGCATCTAACTACC }\end{array}$ & Reverse : CtermVP2 until Nhel (MNV-1) \\
\hline
\end{tabular}

third PCR amplification reaction, $\mathrm{AY}_{4}$ and $\mathrm{AY}_{5}$ were used as the forward and reverse primers, respectively; and the Nhe1 restriction site was included (bold). The first OE-PCR utilised the $5052 \mathrm{~F}$ and $\mathrm{AY}_{3}$ primers with the templates being a combination of the first and the second PCR amplification products. The resulting amplicon was then overlapped with the third PCR amplification product as the second OEPCR employed the $5 \mathrm{O}_{5} \mathrm{~F}$ and $\mathrm{AY}_{5}$ primers to generate the full short strand of DNA.

\section{Polymerase chain reaction (PCR)}

Two PCR amplifications were carried out with the reaction mixture totaling $100 \mu \mathrm{L}$ using the Q5 Hot Start High Fidelity DNA Polymerase according to the manufacture's instructions (New England Biolabs, MA, USA). In the first reaction, the DNA template used was the wt MNV-1 cDNA clone (pT7: MNV 3'Rz), whereas in the second reaction, it was a pNL1.3 plasmid that contained the NanoLuc ${ }^{\mathrm{TM}}$ Luciferase gene (Promega Pte. Ltd., WI, USA). The first OE-PCR was performed using the high fidelity enzyme KOD Hot Start DNA polymerase according to the manufacture's instruction (Merck, USA). The DNA templates in the first OE-PCR were purified DNA amplicons from the first and second PCR reactions. The third PCR was carried out utilising the pNL1.3 as a template. The second OE-PCR was performed employing the purified overlapping DNA amplicons (the first OE-PCR DNA amplicon and the third PCR DNA amplicon). All the above mentioned processes are simplified in Figure 1. The second OE-PCR produced a full short DNA strand covering the DNA sequence of the C-terminal
VP1-secNluc-2A-VP2-Nhe1 (Figure 1). This final DNA amplicon was subjected to agarose gel purification for further use in the cloning process.

\section{Cloning of NanoLuc ${ }^{\mathrm{TM}}$ Luciferase gene into $M N V-1$ cDNA Clone}

\section{Restriction enzyme reaction}

All plasmids (MNV-1 cDNA clones; wt, F/S and $\Delta \mathrm{NS}_{7}$ ) and inserts (purified DNA amplicons of the second OE-PCR) were subjected to restriction enzyme reactions utilising SacII and NheI according to the manufacturer's protocol (NEB, USA). The reaction mixtures were incubated at $37^{\circ} \mathrm{C}$ for 2 hours. Next, agarose gel electrophoresis was performed to produce a purified 3' and 5' overhang insert and vectors for ligation reactions.

\section{Ligation reaction}

Ligation reaction mixtures were set up by mixing a linearised vector and insert with a ligation enzyme and buffers according to the manufacturer's instructions (NEB ligation kit, MA, USA). The mixtures were then incubated at room temperature for 15 minutes before being transformed into competent bacterial cells.

\section{Transformation and positive colony screening}

Transformations were performed by mixing $5 \mu \mathrm{L}$ of each of the ligation mixtures with competent $E$. coli cells (DH5alpha). This was followed by shaking and incubating the mixtures at $37^{\circ} \mathrm{C}$ for 45 minutes. The transformants were then plated on Luria Bertani (LB) agar plates containing $100 \mu \mathrm{g} / \mathrm{mL}$ ampicillin. The plates 
were incubated overnight at $37^{\circ} \mathrm{C}$; and positive clones were then screened using colony PCR before being subjecting to bacterial culture propagation to achieve plasmid DNA extraction and purification.

\section{Large scale production of plasmid DNA}

Positive colonies with inserts of every backbone of MNV-1 cDNA clones were selected for large-scale bacterial production. Plasmid DNA extraction and purification utilising midi preparation kit was performed for each of the bacterial cultures from the positive colonies according to the manufacturer's instructions (Promega, USA). To verify the inserts, all the purified plasmids were subjected to restriction enzyme analysis; and the concentration of each plasmid was estimated using a Nanodrop Spectrophotometer 2000 (Thermo, USA). Lastly, the plasmids were sent to a DNA sequencing service.

\section{Result and Discussion}

\section{Construction of NanoLuc ${ }^{\mathrm{TM}}$ Luciferase-} Tagged Replicon of $M N V$-1

As described in the methodology section regarding the construction of the replicon, a series of five PCR reactions were successfully

\section{SERIES OF POLYMERASE CHAIN REACTIONS}

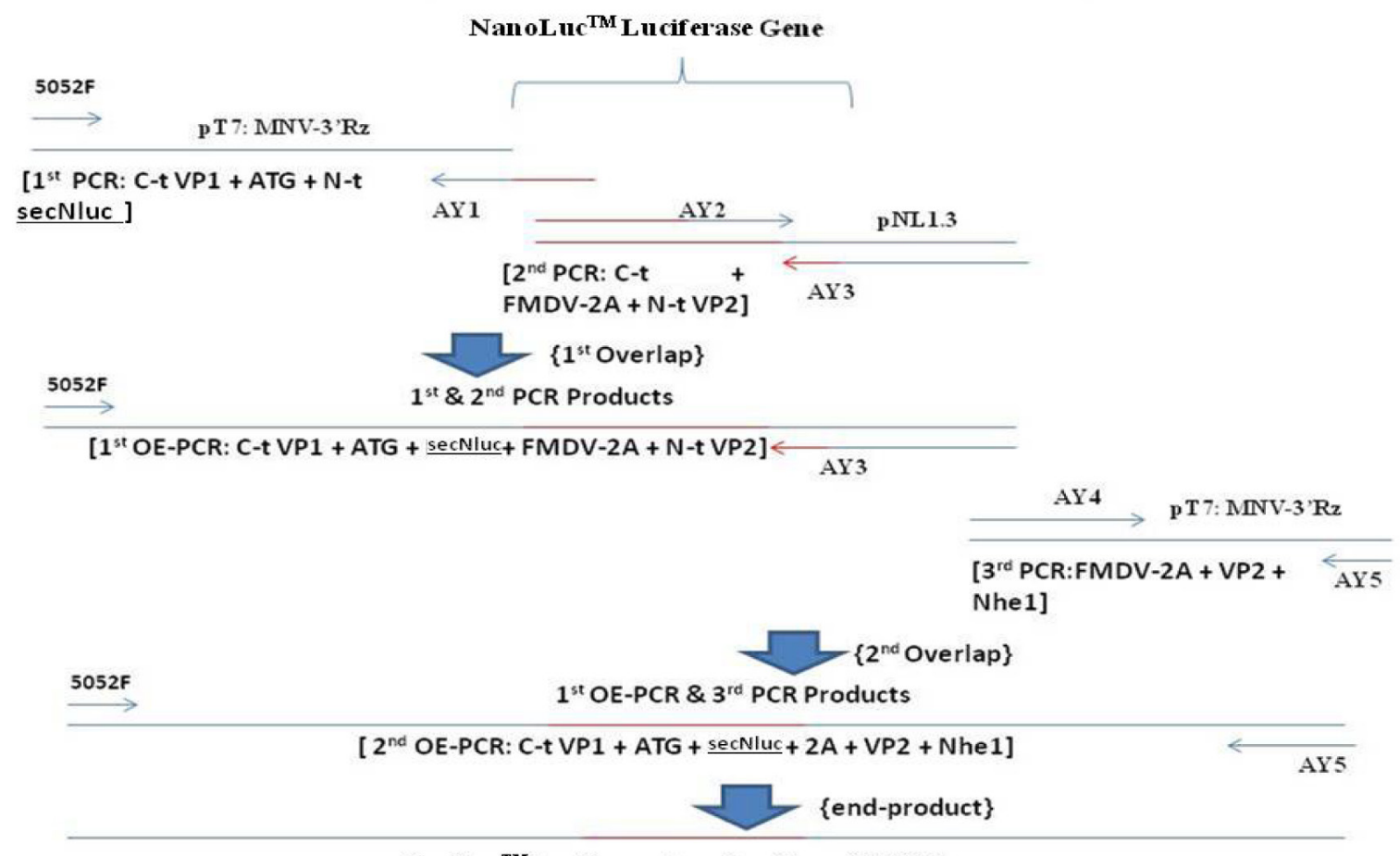

NanoLuc ${ }^{\text {TM }}$ Luciferase - tagged replicon of MINV-1

Figure 1. Schematic diagram illustrating the series of PCR reactions involved in the design and construction of the NanoLuc ${ }^{\mathrm{TM}}$ Luciferase-tagged replicon of MNV-1. The first PCR reaction employed $5052 \mathrm{~F}$ and $\mathrm{AY} 1$ as the forward and reverse primers, respectively, and the MNV-1 cDNA clone (pT7: MNV 3'Rz) as a template. To amplify the secNluc gene from pNL1.3, the second PCR reaction utilised $\mathrm{AY} 2$ and $\mathrm{AY}_{3}$ as the forward and reverse primers, respectively. The third PCR reaction utilised the MNV-1 cDNA clone as the template and $\mathrm{AY}_{4}$ and $\mathrm{AY}_{5}$ as the forward and reverse primers, respectively. The first OE-PCR reaction used the purified DNA amplicons from the first and second PCR reactions as templates with $5052 \mathrm{~F}$ being the forward primer and $\mathrm{AY}_{3}$ being the reverse primer. Finally, the second OE-PCR reaction used the purified DNA amplicons from the first OE-PCR reaction and the purified DNA amplicons from the third PCR reaction as templates, with $5052 \mathrm{~F}$ and $\mathrm{AY}_{5}$ being the forward and reverse primers, respectively. Consequently, a full short DNA strand was produced covering the DNA sequence of the C-terminal VP1 of MNV-1 followed by MNV-1 secNluc, FMDV2A and VP2, and the Nhe1 restriction site 
carried out, including two OE-PCR reactions. The final product was obtained from the second OE-PCR reaction with the amplicon size being 3017 base pairs (bp) (Figure 2). The main purpose of OE-PCR was to introduce the NanoLuc $^{\mathrm{TM}}$ Luciferase gene and FMDV-2A sequences between the VP1 and VP2-encoded genes at the cDNA clone representing $\mathrm{MNV}-1$ genome (Figure 3). This region, which falls into the subgenomic RNA (SgRNA) region of MNV1 full genome was strategically targeted because it plays a role in MNV-1 replication. During viral RNA replication, MNV-1 subgenomic RNA (SgRNA) was synthesised in abundance compared to its full-length RNA genome at a later stage of viral RNA replication. This observation was typically used as a signal for successful $\mathrm{MNV}-1$ replication in the infected cells. Another reason for such a construction strategy was the connection with viral SgRNA translational strategy, which employs a termination-initiation mechanism that causes the separation of translated VP1 and NanoLuc ${ }^{\mathrm{TM}}$ Luciferase proteins $(2,23)$. Perhaps, in this case, the resulting reporter-tagged cDNA was able to produce $\mathrm{MNV}-1$ viral particles through an inherent viral recovery system.

Due to some unique characteristics as compared with other well-established typical reporter genes like Renilla Luciferase, Firefly Luciferase, Green Fluorescent Protein (GFP) and mCherry, the NanoLuc ${ }^{\mathrm{TM}}$ Luciferase

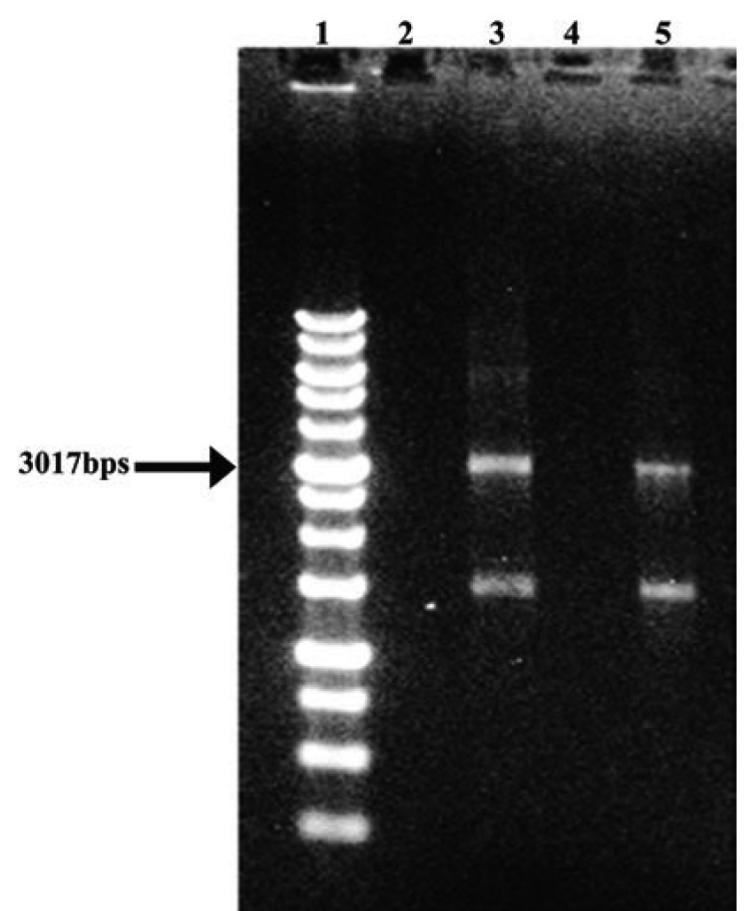

Figure 2. Gel electrophoresis showing a second overlapping-extension PCR (OE-PCR) product prior to gel purification. The bands on the farthest left side belong to a $1-\mathrm{kb}$ DNA ladder (Promega, USA). The third and fifth lanes show the second OE-PCR product with the amplicon size being 3017 bps (top band-red circle)

PT7: MNV-NL2A-VP2-3'Rz

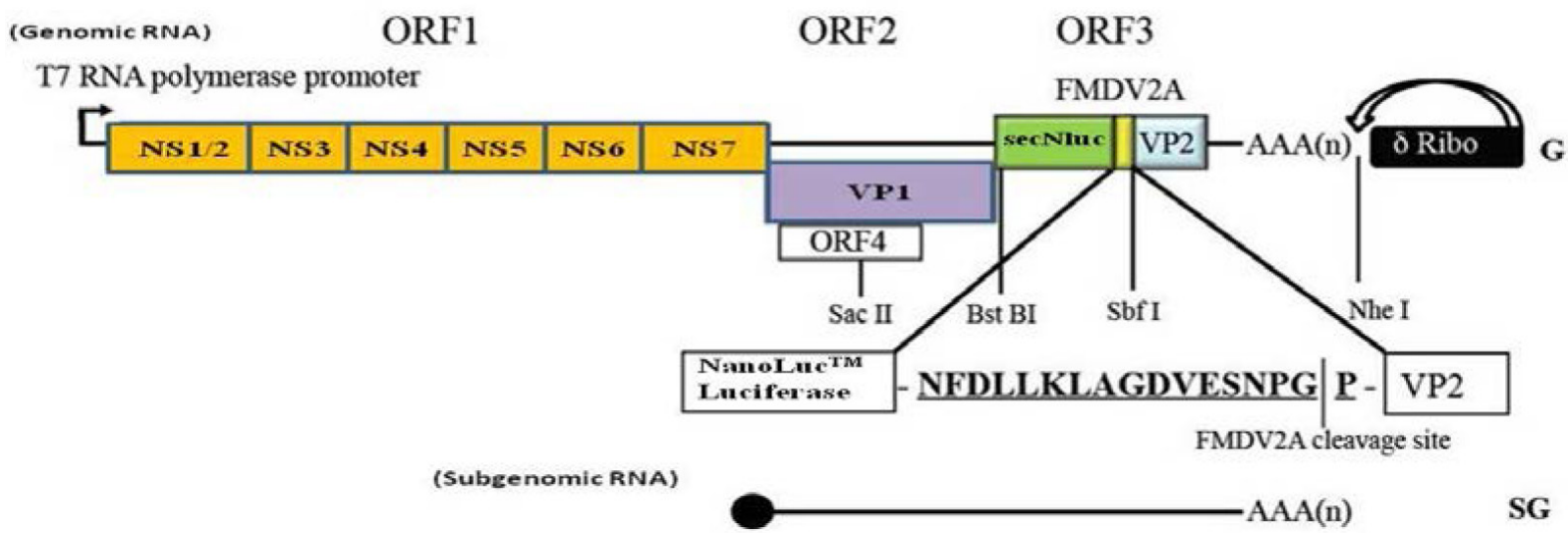

Figure 3. Schematic representation of MNV-1 genomic and subgenomic RNA in the constructed NanoLuc $^{\mathrm{TM}}$ Luciferase-tagged replicon cDNA. The NanoLuc ${ }^{\mathrm{TM}}$ Luciferase (secNluc) gene and the FMDV2A sequence were introduced between ORF2 (VP1) and ORF3 (VP2) in the construct 
gene was deemed more favorable for our MNV-1 replicon system. One of these unique characteristics was the relatively small gene size of NanoLuc ${ }^{\mathrm{TM}}$ Luciferase (597 bp). The insertion of big exogenous sequences into a viral genome is known to hinder viral replication. Furthermore, it may become a major limiting factor contributing to the loss of the function of the reporter gene after multiple rounds of replication during the infection phase. This is due to the generally small genome size of viruses $(22,24)$. In our experiment, the NanoLuc ${ }^{\mathrm{TM}}$ Luciferase reporter protein was also deemed more advantageous as it had been shown to offer superior luminescence in luciferase assays, resulting in higher detection sensitivity when observing MNV-1 replication, particularly during the characterisation stage $(25,26)$.

Another advantage of employing the NanoLuc ${ }^{\mathrm{TM}}$ Luciferase gene for the construction of our reporter-tagged replicon cDNA is that the resulting translated protein was to be expressed as a secreted protein through the secretion signal, interleukin-6 (IL6). In this respect, the mammalian cells transfected with the reportertagged cDNA did not need to be lysed to perform luciferase assays; and the cell culture media could be sampled straight away to enable luciferase detection. This mechanism provided a high-throughput platform to observe MNV-1 replication in vitro. Conversely, a Renilla-tagged replicon system would have necessitated the lysis of the transfected mammalian cells to collect the reporter protein (non-secreted protein) needed for luciferase testing (21).

In the construction stage, the FMDV-2A sequence was inserted between the secNluc gene and the VP2 region. The FMDV-2A sequence is widely used in literature to produce two proteins from a single RNA. In this study, NanoLuc ${ }^{\mathrm{TM}}$ Luciferase and VP2 genes were co-translated and concurrently cleaved in a 2A cleavage mechanism to release the VP2 protein from NanoLuc ${ }^{\mathrm{TM}}$ Luciferase. The separation of the translated NanoLuc ${ }^{\mathrm{TM}}$ Luciferase and VP2 proteins occurred by way of ester-linkage hydrolysis of glycine and proline monomers at the C-terminal of the amino acids encoded by FMDV-2A, leaving proline as the first amino acid residue at the N-terminal of VP2. Therefore, in our replicon construct, the translational products of SgRNA comprised of VP1, NanoLuc ${ }^{\mathrm{TM}}$ Luciferase-2A and VP2 proteins, with NanoLuc ${ }^{\mathrm{TM}}$ Luciferase being secreted out from the host cells (Figure 4) $(27,28)$.

\section{Cloning of NanoLuc ${ }^{\mathrm{TM}}$ Luciferase Gene into $M I N V-1$ cDNA Clone}

The cloning process began with restriction enzyme reactions (SacII and NheI) involving an insert (purified amplicon from the second OE-

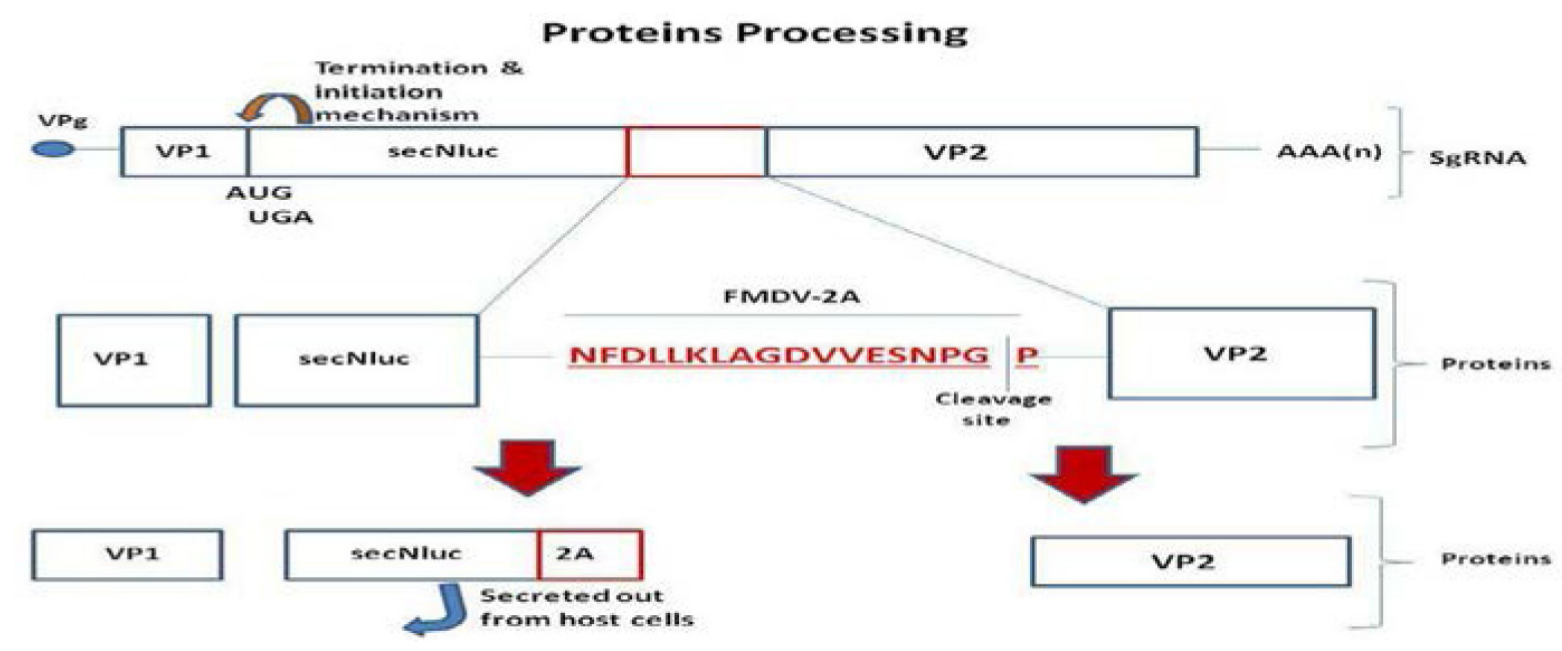

Figure 4. A schematic diagram of protein processing. The release of NanoLuc ${ }^{\mathrm{TM}}$ Luciferase proteins from VP1 is achieved using a ribosome-based termination-initiation mechanism at the overlapping region of VP1 stop codon (UGA) and secNluc start codon (AUG). The release of NanoLuc ${ }^{\mathrm{TM}}$ Luciferase from VP2, however, occurs in a 2A cleavage mechanism at the C-terminal of the amino acid sequences of NanoLuc ${ }^{\mathrm{TM}}$ Luciferase-2A, which involves the hydrolysis of ester linkages between glycine and proline. The translation of this multicistronic SgRNA produces three proteins as follows: VP1, NanoLuc ${ }^{\mathrm{TM}}$ Luciferase-2A and VP2 
PCR reaction) and vectors (MNV-1 cDNA clones; wt, $\mathrm{F} / \mathrm{S}$ and $\Delta \mathrm{NS} 7$ ). As analysed using agarose gel electrophoresis, the complete plasmid reactions produced two distinct DNA bands with the size of 2292 and $10168 \mathrm{bp}$, whereas the complete insert reaction produced the expected DNA band with the size of $2292 \mathrm{bp}$ (Figure 5A and Figure 5B). DNA bands with the size of $10168 \mathrm{bp}$ (vectors) and 2292 bp (inserts) were subjected to gel purification and used in ligation reactions. This was followed by cells transformation into competent bacterial cells; positive clones screening; and large-scale production of each cloned plasmid. Confirmation of clones was carried out using restriction enzyme analysis. The purified plasmids were subjected to restriction enzyme (SacII and NheI) reactions to release inserts of the correct size (2292 bp) (Figure 6). Finally, the large-scale preparation of each plasmid resulted in a high yield of pure cDNA clones as determined using Nanodrop Spectrophotometer 2000 (Table 2). DNA sequencing revealed that all insertions were in the correct order (data not shown).

Three mutant plasmid backbones of the MNV-1 cDNA clone were used in this study as follows: (1) wild-wt, (2) F/S and (3) $\Delta \mathrm{NS} 7$. The plasmids with $\mathrm{F} / \mathrm{S}$ and $\Delta \mathrm{NS} 7$ mutants were derived from the same parental wt MNV-1 cDNA backbone (pT7: MNV 3'Rz). The wt MNV-1 cDNA backbone carried out the full-length viral genome wt sequence from the prototype virus (MNV-1

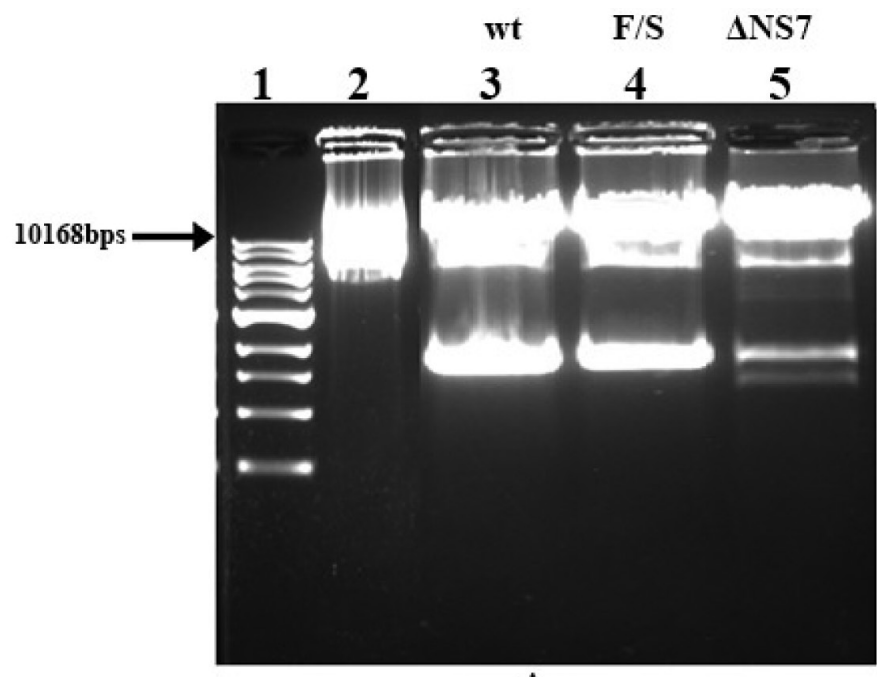

A

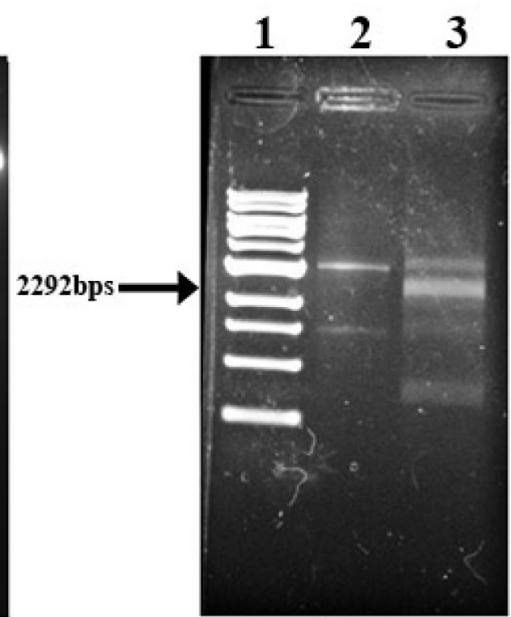

B

Figure 5. (A) Gel electrophoresis following restriction enzyme analysis of three backbones of MNV-1 cDNA clone vectors. The bands on the farthest left side belong to a 1-kb DNA ladder (NEB, USA). The second lane shows the undigested MNV-1 cDNA clone (11810 bps). The third lane shows the digested MNV-1 wt, whereas the fourth lane shows the digested MNV-1 F/S. The fifth lane shows the digested MNV-1 $\Delta$ NS7. (B) Gel electrophoresis following the digestion of the insert (2nd OE-PCR product). The first lane from the left shows a 1-kb DNA ladder (NEB, USA). The second lane shows the undigested insert, whereas the third lane shows the digested insert (red circle)

Table 2. Concentration of DNA samples of every cDNA backbone after DNA ethanol precipitation as measured using Nanodrop Spectrophotometer 2000.

\begin{tabular}{rlccccc} 
No. & \multicolumn{1}{c}{ Experimental sample } & $\begin{array}{c}\text { Concentration } \\
(\mathbf{n g} / \boldsymbol{\mu L})\end{array}$ & $\begin{array}{c}\text { Ratio } \\
(\mathbf{2 6 0 / 2 8 0})\end{array}$ & $\begin{array}{c}\text { Ratio } \\
(\mathbf{2 6 0 / 2 3 0})\end{array}$ & $\begin{array}{c}\text { Sample } \\
\text { Type }\end{array}$ \\
$\mathbf{1}$ & pT7:MNV-secNluc2A-VP2-3'Rz $(\mathrm{wt})$ & 424.2 & 1.83 & 2.10 & DNA \\
$\mathbf{2}$ & pT7:MNV-secNluc2A-VP2-3'Rz (F/S) & 1077.2 & 1.78 & 1.94 & DNA \\
$\mathbf{3}$ & pT7:MNV-secNluc2A-VP2-3'Rz $(\Delta \mathrm{NS} 7)$ & 208.8 & 1.87 & 1.99 & DNA \\
\hline
\end{tabular}




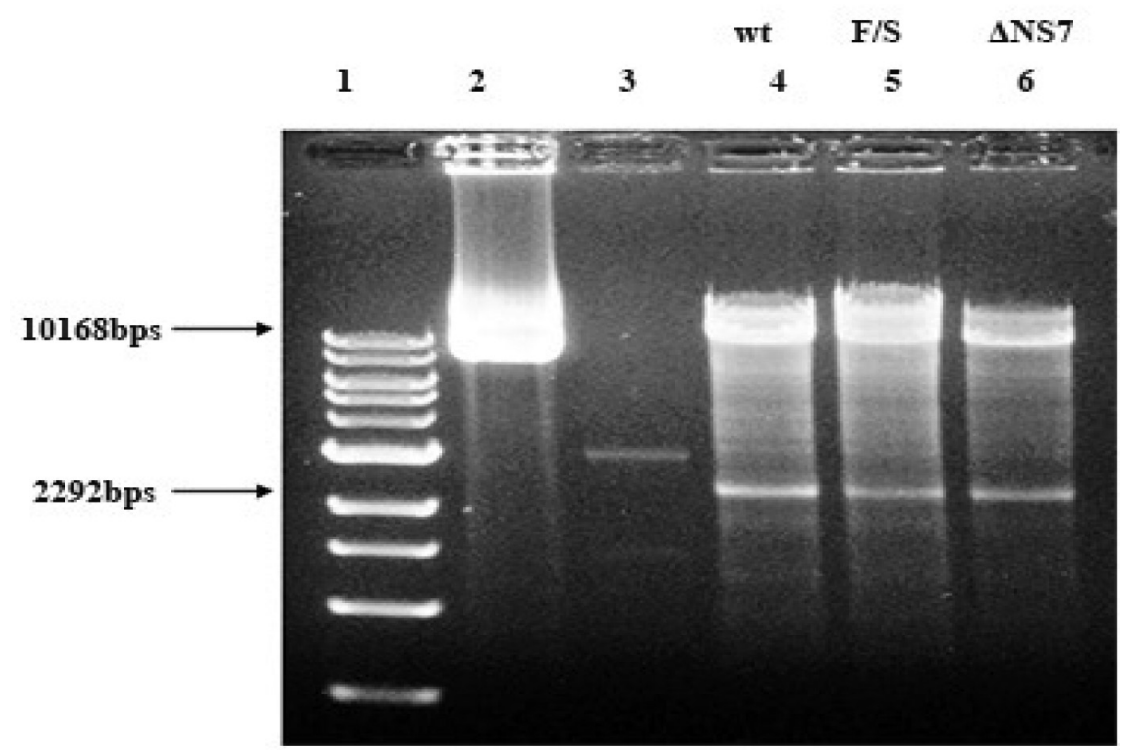

Figure 6. Gel electrophoresis following restriction enzyme analysis of cloned cDNA from positive clones. Three backbones of MNV-1 cDNAs; wt, F/S and $\Delta \mathrm{NS} 7$ were obtained after largescale production and purification (midiprep). The bands on the farthest left side belong to a 1 -kb DNA ladder (NEB, USA). The second and third lanes represent the undigested MNV$1 \mathrm{cDNA}$ clone (1181obps) and the second OE-PCR insert product (3017bps), respectively. The fourth, fifth and sixth lanes show the digested cloned cDNA of pT7. MNV-secNluc2AVP2-3'Rz (wt); pT7. MNV-secNluc2A-VP2-3'Rz (F/S); and pT7. MNV-secNluc2A-VP2-3'Rz $(\triangle \mathrm{NS} 7)$, respectively. Successful restriction enzyme digestion released vectors (10168bps) and inserts (2292bps) of the correct size.

CW.1). To generate the F/S backbone, genetic modification of viral genome was performed, specifically involving the $\mathrm{NS}_{7}$ gene in ORF1. A single nucleotide insertion into the gene created a premature translation termination signal. This frame shift mutation resulted in incomplete translation of the amino acid sequence of MNV1 RNA-dependent RNA polymerase (RdRp), leading to a short and non-functional RdRp. This made the virus unable to undergo genome replication; and no SgRNA was produced. The third backbone in this study was a $\triangle \mathrm{NS} 7$ mutant whereby genetic modification techniques (sitedirected mutagenesis) were used to introduce MNV-1 NS7 gene sequences encoding amino acids of RdRp's active site. The modification resulted in a coding change from GDD to GAA motif residues at the amino acid sequence of RdRp's active site (29). Consequently, $\Delta \mathrm{NS} 7$ cDNA produced an inactive viral RdRp. Both $\mathrm{F} / \mathrm{S}$ and $\triangle \mathrm{NS} 7$ cDNA strands may provide important control points in the reporter-tagged replicon system to thoroughly dissect the stages of norovirus replication based on multiple-level luciferase signal generation.

\section{Conclusion}

This work successfully produced a reportertagged replicon system suitable for MNV-1 in vitro replication research, with the NanoLuc ${ }^{\mathrm{TM}}$ Luciferase gene serving as the reporter gene. Due to the unique and elegant features of this reporter gene, the produced reportertagged cDNA clones may potentially provide a robust experimental assay to study viral RNA replication in vitro. The results of the present study warrant further characterisation of reporter gene translational effectiveness with relation to the three different backbones of MNV1 cDNA clones. The newly developed reportertagged replicon system can potentially provide an efficient tool for researchers to investigate different aspects of norovirus replication.

\section{Acknowledgements}

The present study was supported by a Malaysian Ministry of Higher Education (MOHE) research grant (No. 1001. CIPPT.811233). The authors would like to 
express their gratitude to all the staff at the Advanced Medical and Dental Institute (AMDI), Universiti Sains Malaysia (USM) for providing the necessary assistance and facilities. Special thanks to Professor Ian Goodfellow (University of Cambridge) for supplying the plasmids.

\section{Conflicts of Interest}

The authors declare no conflict of interest regarding the publication of this manuscript. Furthermore, the funding parties had no role in the design of the study; data collection, analysis and interpretation; the writing of the manuscript or the decision to publish the results.

\section{Authors' Contributions}

Conception and design: MKA, MAY

Analysis and interpretation of the data: MKA, MAY

Drafting of the article: MKA, YMT, MAA, ISI, NZ

Critical revision of the article for important intellectual content: YMT, MAA, DAS, RM

Final approval of the article: DAS, RM, MAY

Statistical expertise: ISI, NZ

Administrative, technical, or logistic supports: MAY

\section{Correspondence}

Dr Muhammad Amir Yunus

Deputy Chair for IBSC2018

Head of the Scientific Committee for IBSC2018

BSc (Universiti Sains Malaysia), PhD (Imperial College

London)

Infectomics Cluster,

Advanced Medical \& Dental Institute,

Universiti Sains Malaysia, Bertam,

13200 Kepala Batas,

Pulau Pinang, Malaysia.

Tel: 6045622560

H/P: +6 0194441252

Fax: 6045622349

E-mail: amiryunus@usm.my

\section{References}

1. Hutson AM, Atmar RL, Estes MK. Norovirus disease: changing epidemiology and host susceptibility factors. Trends in Microbiology. 2004;12(6):279-287.

2. Thorne LG, Goodfellow IG. Norovirus gene expression and replication. $J$ Gen Virol. 2014;95(2):278-291
3. Kim MJ, Kim Y-J, Lee JH, Lee JS, Kim JH, Cheon DS, et al. Norovirus: a possible cause of pneumatosis intestinalis. J Pediatr Gastroenterol Nutr. 2011;52(3):314-318.

4. Turcios-Ruiz RM, Axelrod P, John KS, Bullitt E, Donahue J, Robinson N, et al. Outbreak of necrotizing enterocolitis caused by norovirus in a neonatal intensive care unit. $J$ Pediatr. 2008;153(3):339-344.

5. Chen S-Y, Tsai C-N, Lai M-W, Chen C-Y, Lin K-L, Lin T-Y, et al. Norovirus infection as a cause of diarrhea-associated benign infantile seizures. Clinical Infectious Diseases. 2009;48(7):849855 .

6. Bartolini L, Mardari R, Toldo I, Calderone M, Battistella P, Laverda A, et al. Norovirus gastroenteritis and seizures: an atypical case with neuroradiological abnormalities. Neuropediatrics. 2011;42(4):167-169.

7. Chan CM, Chan CW, Ma CK, Chan HB. Norovirus as cause of benign convulsion associated with gastro-enteritis. $J$ Paediatr Child Health. 2011;47(6):373-377.

8. Obinata K, Okumura A, Nakazawa T, Kamata A, Niizuma T, Kinoshita K, et al. Norovirus encephalopathy in a previously healthy child. The Pediatric Infectious Disease Journal. 2010;29(11):1057-1059.

9. Brown D, Gray J, MacDonald P, Green A, Christopher G, Glass R, et al. Outbreak of acute gastroenteritis associated with Norwalk-like viruses among British military personnel-Afghanistan, May 2002. Morbidity and Mortality Weekly Report. 2002;51(22):477-479.

10. Mattner F, Sohr D, Heim A, Gastmeier P, Vennema H, Koopmans M. Risk groups for clinical complications of norovirus infections: an outbreak investigation. Clinical Microbiology and Infection. 2006;12(1):69-74.

11. Bok K, Green KY. Norovirus gastroenteritis in immunocompromised patients. New England Journal of Medicine. 2012;367(22):2126-2132.

12. Capizzi T, Makari-Judson G, Steingart R, Mertens WC. Chronic diarrhea associated with persistent norovirus excretion in patients with chronic lymphocytic leukemia: report of two cases. $B M C$ Infectious Diseases. 2011;11:131. https://doi. org/10.1186/1471-2334-11-131 
13. Wobus CE, Karst SM, Thackray LB, Chang K-O, Sosnovtsev SV, Belliot G, et al. Replication of norovirus in cell culture reveals a tropism for dendritic cells and macrophages. PLoS Biol. 2004;2(12):e432.

14. Duizer E, Schwab KJ, Neill FH, Atmar RL, Koopmans MP, Estes MK. Laboratory efforts to cultivate noroviruses. $J$ Gen Virol. 2004;85(1):79-87.

15. Karst SM, Wobus CE, Lay M, Davidson J, Virgin HW. STAT1-dependent innate immunity to a Norwalk-like virus. Science. 2003;299(5612):1575-1578.

16. Sosnovtsev SV, Belliot G, Chang K-O, Prikhodko VG, Thackray LB, Wobus CE, et al. Cleavage map and proteolytic processing of the murine norovirus nonstructural polyprotein in infected cells. $J$ Virol. 2006;80(16):7816-7831.

17. McFadden N, Bailey D, Carrara G, Benson A, Chaudhry Y, Shortland A, et al. Norovirus regulation of the innate immune response and apoptosis occurs via the product of the alternative open reading frame 4. PLoS Pathog. 2011;7(12):e1002413. https://doi.org/10.1371/ journal.ppat.1002413

18. Simmonds P, Karakasiliotis I, Bailey D, Chaudhry Y, Evans DJ, Goodfellow IG. Bioinformatic and functional analysis of RNA secondary structure elements among different genera of human and animal caliciviruses. Nucleic Acids Research. 2008;36(8):2530-2546.

19. Bailey D, Karakasiliotis I, Vashist S, Chung LMW, Reese J, McFadden N, et al. Functional analysis of RNA structures present at the 3 ' extremity of the murine norovirus genome: the variable polypyrimidine tract plays a role in viral virulence. $J$ Virol. 2010;84(6):2859-2870.

20. Hardy ME. Norovirus protein structure and function. FEMS Microbiology Letters. 2005;253(1):1-8.

21. Yunus MA, Chung LMW, Chaudhry Y, Bailey D, Goodfellow I. Development of an optimized RNAbased murine norovirus reverse genetics system. $J$ Virol Methods. 2010;169(1):112-118.
22. Sun C, Gardner CL, Watson AM, Ryman KD, Klimstra WB. Stable, high-level expression of reporter proteins from improved alphavirus expression vectors to track replication and dissemination during encephalitic and arthritogenic disease. J Virol. 2014;88(4):20352046.

23. Katayama K, Hansman G, Oka T, Ogawa $\mathrm{S}$, Takeda $\mathrm{N}$. Investigation of norovirus replication in a human cell line. Arch Virol. 2006;151(7):1291-1308.

24. England CG, Ehlerding EB, Cai W. NanoLuc: a small luciferase is brightening up the field of bioluminescence. Bioconjug Chem. 2016;27(5):1175-1187.

25. Loh JM, Proft T. Comparison of firefly luciferase and NanoLuc luciferase for biophotonic labeling of group A Streptococcus. Biotechnology Letters. 2014;36(4):829-834.

26. Tran V, Moser LA, Poole DS, Mehle A. Highly sensitive real-time in vivo imaging of an influenza reporter virus reveals dynamics of replication and spread. $J$ Virol. 2013;87(24):13321-13329.

27. Doronina VA, Wu C, de Felipe P, Sachs MS, Ryan MD, Brown JD. Site-specific release of nascent chains from ribosomes at a sense codon. Molecular and Cellular Biology. 2008;28(13):4227-4239.

28. Doronina VA, de Felipe $\mathrm{P}$, Wu C, Sharma P, Sachs MS, Ryan MD, et al. Dissection of a co-translational nascent chain separation event. Biochemical Society Transactions. 2008;36(4):712-716.

29. Subba-Reddy CV, Goodfellow I, Kao CC. VPgprimed RNA synthesis of norovirus RNAdependent RNA polymerases by using a novel cell-based assay. J Virol. 2011;85(24):1302713037 .

30. Yunus MA, Lin X, Bailey D, Karakasiliotis I, Chaudhry Y, Vashist S, et al. The murine norovirus core subgenomic RNA promoter consists of a stable stem-loop that can direct accurate initiation of RNA synthesis. $J$ Virol. 2015;89(2):1218-1229. 